\title{
Is AI Forcing the Reincarnation of Quality?
}

\section{Christopher Wickenden}

Fresenius University of Applied Sciences, Department SKIP Institute for Applied Digital Visualization, Cologne, Germany
${ }^{*}$ Corresponding author

Christopher Wickenden, Fresenius University of Applied Sciences, Department SKIP Institute for Applied Digital Visualization, Cologne, Germany

Submitted: 06 Oct 2020; Accepted: 21 Oct 2020; Published: 30 Oct 2020

The consequences could possibly lead to a new form of uncontrolled perceived quality, which is cunningly disguised by AI as dictated behavior.

\section{Theory}

Let us assume we currently rely on a separate balance of 2 tracks (fact and Instinct) of decision- making influences.

This will most likely lead to a human natural reaction of decisionmaking (Figure 1).

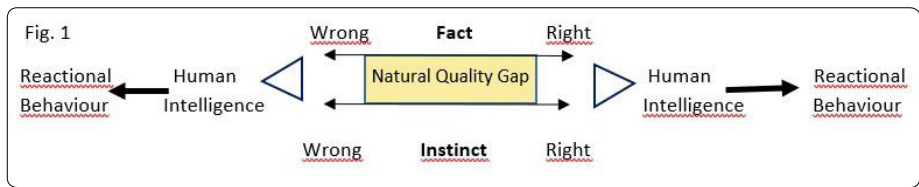

Figure 1: Whatever the decision - right or wrong, it is based on fact or instinct therefore, naturally human and an exceptionally natural form of perceived quality, hence, the gap between the tracks.

Let us also assume with the growing interrelation of fact and instinct through algorithmic iteration and correction, the decisions we make in the future could be deeply influenced without individual human natural consideration. Therefore, the evaluated algorithmic result is an artificial decision, based on gathered volume and relation of fact and instinct, without a wrong direction. This would develop over generations and become an evolutionary impact on the way we think and make decisions. The outcome would inevitably be an artificially dictated behavior, without any considered alternative natural decision (Figure 2).

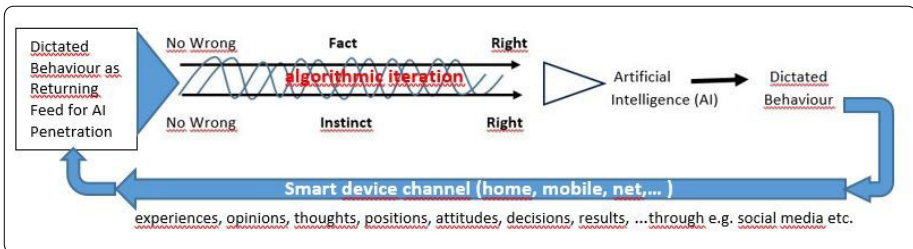

Figure 2: The quality gap diffuses and disappears, due to continuous interrelation exchange of algorithmic iteration. future behavior and how we may lose our natural instinct through the penetrating determination of algorithmic correction. 
Eventually it becomes one track and we have no alternative. The collected dictated behavior flows back through the smart device channel as feed for further interpretations of personal information and instinctive response. It overwrites the two tracks - the process repeats.

Figure 2 also assumes, that we supply data from which an algorithm can feed. In terms of facts, this is no challenge. But how can we deliver sufficient data of instinctual reaction and decisions. How can mental access to our personal thoughts and beliefs be granted?

We see how smartphones offer all access to our very personal thoughts, our planning, scheduling, destinations, interests, preferences, opinions, ... and the list goes on. We supply vital and detailed information on our behavior and release many important details over social media on what we think and how we react.

We, the millions, offer voluntarily the most intimate informationup for grabs. So the question may not necessarily be how AI gains mental access, but how is the available data interpreted accordingly?

And furthermore, who runs this deal we involuntarily (voluntarily?) agree to? Given the growth of social media, the worrying enthusiasm with which we release our personal and intimate daily life to the rest of the world, we obviously believe AI is a trustworthy helper, whoever installs it.

\section{AI - The Spoiler or Helper?}

This is probably the most important question and this is probably an issue in which we have to focus particular concern. The various ethics and economic guidelines currently being developed and published to preserve safety and regulated data protection laws for the benefit of our societies around the world, are exactly what is needed.
However, no matter what company, economy, society or political understanding might be the center of controversy for abusing these regulations - we must also realize, that we, mankind, apparently enjoy the comfort of living a life in which we can pass on responsibility, turn away when others suffer, be led by the majority and just reach out for anything that might improve our standard of living or offer us wealth, despite the consequences.

AI has so much power to spoil us, but even more it has the potential to help us.

\section{Food for thought}

This might be the natural evolutionary design of mankind. It sometimes seems frightening; it sometimes seems relieving. This approach on the subject of AI as a tool for quality, may give food for thought. I am open to the technological developments we currently pursue and realize how we can benefit from the extremely dynamic process we are driving constantly to gain the evidence and results we need to bring up discussions and questions on ethical and moral destinations. I also accept the possibility that what we are currently heading to, is a part of our natural evolutionary design.

Therefore, we might question ourselves what quality is these days, what was it in the past and what is it in the future? Bearing the current climate change, the mass migrations, growth of uneven share, the pollution of the planet, the covid-19 pandemic and the everlasting search for solutions to the UN 17 sustainability goals, maybe focused AI will offer reincarnated quality of life, far from what we have (instinctively?) believed was quality up to now.
Copyright: (02020 Christopher Wickenden. This is an open-access article distributed under the terms of the Creative Commons Attribution License, which permits unrestricted use, distribution, and reproduction in any medium, provided the original author and source are credited. 